\title{
Increased RCAS1 Expression Is Associated with Advanced Histopathological Stage and Poor Prognosis in Patients with Gastric Adenocarcinoma
}

\author{
Constantinos Giaginis, ${ }^{1,2}$ Themistoclis Efkarpidis, ${ }^{3}$ Paraskevi Alexandrou, ${ }^{1}$ \\ Efstratios Patsouris, ${ }^{1}$ Gregory Kouraklis, ${ }^{3}$ and Stamatios Theocharis ${ }^{1}$ \\ ${ }^{1}$ First Department of Pathology, Medical School, National and Kapodistrian University of Athens, \\ 75 M. Asias steet, Goudi, GR11527 Athens, Greece \\ ${ }^{2}$ Department of Food Science and Nutrition, University of the Aegean, Myrina, Lemnos, Greece \\ ${ }^{3}$ Second Department of Propedeutic Surgery, Medical School, University of Athens, Athens, Greece \\ Correspondence should be addressed to Stamatios Theocharis; statheocharis@yahoo.com
}

Received 29 June 2013; Accepted 23 August 2013

Academic Editor: Sun-Il Hwang

Copyright (C) 2013 Constantinos Giaginis et al. This is an open access article distributed under the Creative Commons Attribution License, which permits unrestricted use, distribution, and reproduction in any medium, provided the original work is properly cited.

\begin{abstract}
Background. The receptor-binding cancer antigen expressed on SiSo cells (RCAS1) is a human tumor-associated antigen that has been considered to play a crucial role in tumor progression by enabling cancer cells to evade immune surveillance. The present study aimed to evaluate the clinical significance of the RCAS1 expression in gastric adenocarcinoma. Material and Methods. RCAS1 protein expression was assessed immunohistochemically on 54 gastric adenocarcinoma tissue samples and was analyzed in relation to clinicopathological parameters, tumor proliferative capacity, and patients' survival. Results. Enhanced RCAS1 expression levels were significantly associated with advanced histopathological stage and presence of organ metastasis $(P=0.0084$ and $P=0.0327)$. Gastric cancer patients with elevated RCAS1 expression levels showed significantly shorter survival times compared to those with low RCAS1 expression (log-rank test, $P=0.0168$ ). In multivariate analysis, histopathological stage and grade of differentiation as well as the RCAS1 expression were identified as independent prognostic factors (Cox regression analysis, $P=0.0204, P=$ 0.0035 , and $P=0.0081$ ). Conclusions. Our data support the evidence that RCAS1 upregulation may contribute to gastric malignant progression, representing a useful biomarker to predict the biological behaviour and prognosis in gastric neoplasia.
\end{abstract}

\section{Background}

The receptor-binding cancer antigen expressed on SiSo cells (RCAS1) was initially recognized by the mouse monoclonal antibody, 22-1-1, which was raised by the immunization of mice with the human uterine cervical adenocarcinoma cell line SiSo [1]. It is a 40-kd type II membrane protein that forms homo-oligomers through its C-terminal coiledcoil structures, whereas it also exists in the soluble form probably by alternative splicing [2]. RCAS1 has currently been considered as a novel tumor-associated antigen that can function as a ligand for a putative receptor that is present on various human cell lines, such as erythroid leukaemia and normal peripheral lymphocytes, inhibiting cell growth and inducing apoptosis $[3,4]$. Thus, RCAS1 upregulation is considered to play a crucial role in tumor progression by enabling cancer cells to evade immune surveillance $[5,6]$.

Gastric cancer is the second largest cause of cancerrelated death worldwide, presenting the higher incidence in Japan and China, lower in Europe, and the lowest in the USA $[7,8]$. Studies on gastric cancer development suggest that genetic predisposition, infection, and diet are parts of a complex interaction [8, 9]. Helicobacter pylori (HP) infection and to a lesser extent smoking have been identified as the main environmental risk factors for gastric cancer $[8,9]$. Chronic inflammation plays important roles in the development of various cancers of the digestive tract, including HPassociated gastric cancer [10]. Notably, several inflammatory mediators have been associated with tumor progression, angiogenesis, and metastasis in gastric cancer [11, 12]. 
In the last few years, RCAS1 protein expression has been described in a variety of human malignancies, including oral, esophageal, breast, thyroid, lung, neurological, pancreatic, colorectal, urothelial, endometrial, cervical, and ovarian carcinomas $[13,14]$. Notably, RCAS1 expression was shown to be associated with important clinicopathological parameters for patients' management and prognosis, being considered as an informative biomarker in several types of tumour malignancy $[13,14]$. However, the available studies so far assessing the clinical significance of the RCAS1 expression in gastric neoplasia remain scarce, and they are restricted to T3 gastric carcinoma cases or they do not concern patients' prognosis [15-17]. In view of the previous considerations, the present study aimed to assess immunohistochemically RCAS1 expression in 54 gastric adenocarcinoma specimens and to evaluate the association of RCAS1 expression levels with clinicopathological parameters, tumor proliferative capacity, and patients' survival.

\section{Materials and Methods}

2.1. Patients. Fifty-four gastric tumoral samples obtained from an equal number of patients, who underwent surgical resection due to gastric cancer at the 2nd Department of Propedeutic Surgery, Medical School, University of Athens, Greece, were consecutively included in this study. The study was approved by the hospital ethics committee, and informed consent was obtained from all participants. None of the patients had received chemotherapy or radiation before surgery. Forty-three $(79.6 \%)$ of the patients were men and $11(20.4 \%)$ were women. The mean age of the patient cohort was $67.33 \pm 8.47$ years (median: 67 years, range: $39-88$ years). Tumors were typed according to Lauren classification as intestinal in $24(44.4 \%)$ and diffuse in 30 (55.6\%) gastric adenocarcinoma cases [18]. Three levels differentiation were used to classify grading as: well in $1(1.9 \%)$, moderately in 24 (44.4\%), and poorly differentiated in 29 (53.7\%) patients. Tumors staging was assessed using the 5th edition of the Tumor, Node, and Metastasis (TNM) system according to the Union Internationale Contra la Cancrum (UICC) and the American Joint Committee on Cancer (AJCC) [19]. The resected tumors were staged as: $\mathrm{T} 1$ in $6(11.1 \%)$; T2 in 15 (27.7\%); T3 in 27 (50.0\%), and T4 in 6 (11.1\%) cases. Nineteen (35.2\%) patients were lymph node negative (N0) and 35 (64.8\%) lymph node positive; N1 in 32 (59.3\%) and N2 in $3(5.6 \%)$ cases. Seven $(13.0 \%)$ patients presented organ metastasis. Patients were followed up for a time interval between 1 to 148 months ( $28.21 \pm 22.63$ months). Thirty $(55.6 \%)$ patients were dead at the time of the last followup.

2.2. Immunohistochemistry. RCAS1 immunostaining was performed on formalin-fixed, paraffin-embedded gastric adenocarcinoma tissue sections using a mouse monoclonal anti-RCAS1 antibody (MBL International Co, Nagoya, Japan). Briefly, $4 \mu \mathrm{m}$ thick tissue sections were dewaxed in xylene and were brought to water through graded alcohols. Antigen retrieval was performed by microwaving slides in $10 \mathrm{mM}$ citrate buffer ( $\mathrm{pH}$ 6.1) for 20 minutes (min) at high power, according to the manufacturer's instructions. To remove the endogenous peroxidase activity, sections were treated with freshly prepared $0.3 \%$ hydrogen peroxide in methanol in the dark, for $30 \mathrm{~min}$, at room temperature. Nonspecific antibody binding was blocked using Sniper, a specific blocking reagent for mouse primary antibodies (Sniper, Biocare Medical, Walnut, Creek, CA, USA) for $5 \mathrm{~min}$. The sections were incubated for 1 hour (h), at room temperature, with the primary antibody against RCAS1, diluted 1:750 in phosphate buffered saline (PBS). After washing three times with PBS, sections were incubated at room temperature with biotinylated linking reagent (Biocare Medical) for $10 \mathrm{~min}$, followed by incubation with peroxidase-conjugated streptavidin label (Biocare Medical) for $10 \mathrm{~min}$. The resultant immune peroxidase activity was developed using a DAB substrate kit (Vector Laboratories, USA) for $10 \mathrm{~min}$. Sections were counterstained with Harris' hematoxylin and mounted in Entellan (Merck, Darmstadt, Germany). Appropriate negative controls were performed by omitting the primary antibody and/or substituting it with an irrelevant antiserum. Pancreatic and thyroid cancer tissue sections with known increased RCAS1 immunoreactivity were used as positive control [20, 21].

2.3. Evaluation of Immunohistochemistry. Immunohistochemical evaluation was performed by counting at least 1000 tumor cells in each case by two independent observers (S.T. and P.A.) blinded to the clinical data, with complete observer agreement. The immunoreactivity of the tumor cells for RCAS1 was scored according to the percentage of RCAS1 positive tumor cells as $0:<5 \%$ of tumor cell positive; 1: $5-24 \%$ of tumor cells positive; 2 : $25-49 \%$ of tumor cells positive; 3: $50-74 \%$ of tumor cells positive; 4: and $75-100 \%$ of tumor cells positive and according to its intensity as 0 : negative staining, 1: mild staining; 2 : intermediate staining; and 3: intense staining. Finally, the expression of RCAS1 was classified as low; if the total score was $\leq 5$ and high; if the total score was $\geq 6$. In this way, we ensure that each group has a sufficient and more homogeneous number of cases in order to be comparable with the other groups [20-22].

2.4. Statistical Analysis. Chi-square test was used to assess the associations of RCAS1 protein expression with clinicopathological variables. Survival curves were constructed using the Kaplan-Meier method and the differences between the curves were compared by the log-rank test. A Cox ProportionalHazards regression model was developed to evaluate the association between the potential prognostic marker and patients' survival. Cox regression analysis was conducted at both univariate and multivariate levels. A $P$ value less than 0.05 was considered the limit of statistical significance. SPSS for Windows software was used for all analyses (SPSS Inc., 2003, Chicago, USA).

\section{Results}

RCAS1 was abundantly expressed in the gastric adenocarcinoma cases examined, presenting both a cytoplasmic and membraneous pattern of staining (Figure 1). All cases were 
found positive for RCAS1, while the mean percentage RCAS1 expression value was $64.5 \%$. The intensity of RCAS1 immunostaining was classified as mild in 4 (7.4\%), moderate in 19 (35.2\%), and intense in 21 (57.4\%) out of 54 gastric adenocarcinoma cases. By applying the immunohistochemical score, $25(46.3 \%)$ cases were found to present low RCAS1 expression, and the remaining 29 (53.7\%) cases showed high expression. Weak cytoplasmic RCAS1 expression was also noted in less than $5 \%$ in normal (nonmalignant) gastric tissues.

In cross-tables, high RCAS1 expression was significantly more frequently observed in gastric adenocarcinoma cases with advanced histopathological stage and presence of organ metastasis (Table $1, P=0.0327$ and $P=0.0084$, resp.). RCAS1 expression did not show statistical significant associations or trends of correlation with the other clinicopathological variables examined (Table $1, P>0.05$ ).

Univariate analysis was performed to assess the strength of the association of each clinicopathological parameter and RCAS1 expression (low versus high) with overall patients' survival. Patients' age, histopathological type, grade of differentiation, disease stage, tumor size, presence of lymph node and organ metastasis, as well as RCAS1 expression were identified as significant prognostic factors of overall patients' survival (Table 2, $P=0.0031, P=0.0003, P<0.001, P=$ $0.001,0.023,0.0021,0.09$, and $P=0.021$, resp.). Kaplan-Meier survival curves indicated that patients with high RCAS1 expressing tumors had significantly shorter survival times compared to those with low RCAS1 expression (Figure 2(a), $\log$-rank test, $P=0.016$ ). The mean survival time in patients presenting high RCAS1 expression was 33.88 months $( \pm 23.19$ months), whereas in those presenting low RCAS1 expression was 23.32 months ( \pm 17.73 months). In multivariate analysis, tumor histopathological grade of differentiation, stage, and RCAS1 expression proved to be independent prognostic factors of overall patients' survival (Table $3, P=0.003, P=$ 0.020 and $P=0.008$, resp.).

Statistical analysis for RCAS1 expression was further performed in each gastric adenocarcinoma histopathological type, separately. In diffuse-type gastric adenocarcinoma, RCAS1 expression was significantly associated with the presence of organ metastasis $(P=0.025)$ and borderlined with histopathological stage $(P=0.078)$. Kaplan-Meier survival curves indicated that diffuse-type gastric adenocarcinoma patients with high RCAS1 expression presented significantly shorter survival times compared to those with low RCAS1 expression (Figure 2(b), log-rank test, $P=0.033$ ). Histopathological stage and RCAS1 expression were identified as significant predicting factors for patients prognosis in multivariate analysis (Cox regression analysis, $P=0.018$ and $P=0.048$, resp.). In intestinal-type gastric adenocarcinoma, RCAS1 expression was not associated with any clinicopathological parameters. Intestinal-type gastric adenocarcinoma patients presenting high RCAS1 expression levels showed shorter survival times compared to those with low levels at a no significant level, though (Figure 2(c), log-rank test, $P=$ $0.225)$.

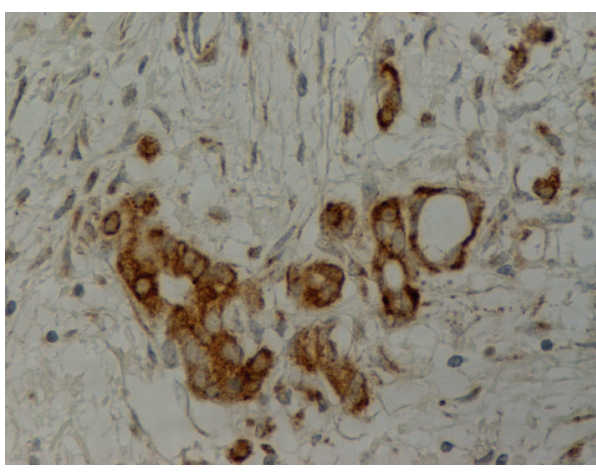

(a)

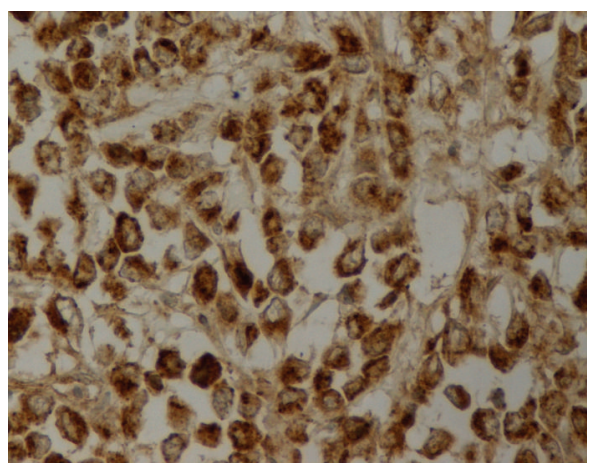

(b)

FIGURE 1: Representative immunostainings for cytoplasmic and membraneous RCAS1 expression in tumor cells of gastric adenocarcinoma. Streptavidin-biotin-peroxidase, DAB chromogen, and Harris hematoxylin counterstain (original magnification $\times 400$ ). (a) Moderately differentiated intestinal-type gastric cancer case and (b) poorly differentiated diffuse-type gastric cancer case.

\section{Discussion}

RCAS1 has been documented to be overexpressed in various tumors, affecting many aspects of cancer biology, such as differentiation, proliferation, invasion, and angiogenesis [13, $14,23]$. Elevated RCAS1 expression has been associated with the malignant state of several tissue types and may play crucial role in tumor progression by enabling cancer cells to evade immune surveillance $[13,14,23]$. The expression of RCAS1 in tumors of the gastrointestinal tract has already been documented; however, its associations with clinicopathological parameters and patients' prognosis in gastric neoplasia remains scarce.

In the present study, all the examined gastric adenocarcinoma cases were found positive for RCAS1. Moreover, 29 (53.7\%) out of the 54 gastric cancer cases showed high RCAS1 expression. Notably, current substantial evidence supported that the fact RCAS1 may be considered as a promising target for future (gene) therapeutic approaches [13, 14, 23]. In this aspect, the increased frequency of high RCAS1 positivity in the examined gastric adenocarcinoma cases reinforces the therapeutic utility of this receptor in gastric cancer chemoprevention and treatment. Importantly, the recent evidence that RCAS1 siRNA suppressed RCAS1 mRNA and protein expression and delayed tumor growth, in vivo, has already 
TABLE 1: Associations of RCAS1 expression with clinicopathological characteristics in 54 patients with gastric adenocarcinoma.

\begin{tabular}{|c|c|c|c|}
\hline \multirow{2}{*}{$\begin{array}{l}\text { Clinicopathological } \\
\text { parameters }(n=54)\end{array}$} & \multicolumn{2}{|c|}{ RCAS1 expression } & \multirow{2}{*}{$P$ value } \\
\hline & Low (\%) & High (\%) & \\
\hline Patients & $25(46.3)$ & $29(53.7)$ & \\
\hline Age & & & 0.8156 \\
\hline$<67.33$ yrs & $12(22.2)$ & $13(24.1)$ & \\
\hline$\geq 67.33$ yrs & $13(24.1)$ & $16(29.6)$ & \\
\hline Gender & & & 0.1961 \\
\hline Men & $18(33.3)$ & $25(46.3)$ & \\
\hline Women & $7(13.0)$ & $4(7.4)$ & \\
\hline Histological grade & & & 0.8156 \\
\hline $\begin{array}{l}\text { Well + moderately } \\
\text { differentiated }\end{array}$ & $12(22.2)$ & $13(24.1)$ & \\
\hline Poorly differentiated & $13(24.1)$ & $16(29.6)$ & \\
\hline pT classification & & & 0.8555 \\
\hline $\mathrm{T} 1$ & $3(5.6)$ & $3(5.6)$ & \\
\hline $\mathrm{T} 2$ & $8(14.8)$ & $7(13.0)$ & \\
\hline T3 & $12(22.2)$ & $15(27.8)$ & \\
\hline $\mathrm{T} 4$ & $2(3.7)$ & $4(7.4)$ & \\
\hline pN classification & & & 0.7434 \\
\hline No & $9(16.7)$ & $10(18.5)$ & \\
\hline N1 & $14(25.9)$ & $18(33.3)$ & \\
\hline $\mathrm{N} 2$ & $2(3.7)$ & $1(1.8)$ & \\
\hline pM classification & & & 0.0084 \\
\hline M0 & $25(46.3)$ & $22(40.7)$ & \\
\hline M1 & $0(0)$ & $7(13.0)$ & \\
\hline pStage & & & 0.0327 \\
\hline I & $6(11.1)$ & $6(11.1)$ & \\
\hline II & $10(18.5)$ & $6(11.1)$ & \\
\hline III & $9(16.7)$ & $9(16.7)$ & \\
\hline IV & $0(0)$ & $8(14.8)$ & \\
\hline Histopathological type & & & 0.2995 \\
\hline Intestinal & $13(24.1)$ & $11(20.4)$ & \\
\hline Diffuse & $12(22.2)$ & $18(33.3)$ & \\
\hline $\begin{array}{l}\text { Ki- } 67 \text { protein } \\
\text { statement }\end{array}$ & & & 0.1588 \\
\hline Below mean value & $9(16.7)$ & $16(29.6)$ & \\
\hline Over mean value & $16(29.6)$ & $13(24.1)$ & \\
\hline
\end{tabular}

unfolded new perspectives for the development of future RCAS1 receptor target inhibitors [24]. Antiestrogens may also employ RCAS1 as a molecular switch to activate immune cytotoxicity against tumor cells [25].

The present study further showed that RCAS1 expression was significantly elevated in gastric cancer patients with advanced histopathological stage and presence of organ metastasis. Elevated RCAS1 expression was also significantly associated with poor patients' prognosis in both univariate
TABLE 2: Association of clinicopathological variables and RCAS1 expression with patients' survival: univariate analysis.

\begin{tabular}{|c|c|c|}
\hline Clinicopathological Variables & $\operatorname{HR}(95 \% \mathrm{CI})$ & $P$ value \\
\hline \multicolumn{3}{|l|}{ Age } \\
\hline$(<67.33 / \geq 67.33$ yrs $)$ & $2.171(1.082-4.037)$ & 0.031 \\
\hline \multicolumn{3}{|l|}{ Gender } \\
\hline (Male/female) & $0.748(0.355-1.601)$ & 0.398 \\
\hline \multicolumn{3}{|l|}{ Histopathological type } \\
\hline (Intestinal/diffuse) & $2.701(1.384-5.301)$ & 0.003 \\
\hline \multicolumn{3}{|l|}{ Histopathological grade } \\
\hline (Well + moderately/poorly) & $5.467(2.512-11.701)$ & $<0.001$ \\
\hline \multicolumn{3}{|l|}{ pT classification } \\
\hline (T1-2/T3-4) & $2.654(1.093-4.340)$ & 0.023 \\
\hline \multicolumn{3}{|l|}{ pN classification } \\
\hline (N0/N1-2) & $2.530(1.181-5.498)$ & 0.021 \\
\hline \multicolumn{3}{|l|}{ pM classification } \\
\hline (M0/M1) & $3.180(1.277-7.645)$ & 0.009 \\
\hline \multicolumn{3}{|l|}{ pStage } \\
\hline$(\mathrm{I}+\mathrm{II} / \mathrm{III}+\mathrm{IV})$ & $3.445(1.782-6.450)$ & 0.001 \\
\hline \multicolumn{3}{|l|}{ RCAS1 expression } \\
\hline (Low/high) & $1.877(0.950-3.459)$ & 0.021 \\
\hline
\end{tabular}

TABLE 3: Multivariate analysis of RCAS1 expression adjusted for patients' age, histopathological type, grade, and stage.

\begin{tabular}{lcc}
\hline Clinicopathological Variables & HR $(95 \%$ CI $)$ & $P$ value \\
\hline $\begin{array}{l}\text { Age } \\
\quad(<67.33 / \geq 67.33 \text { yrs })\end{array}$ & $1.113(1.332-3.132)$ & 0.328 \\
$\begin{array}{l}\text { Histopathological type } \\
\quad \text { Intestinal/diffuse })\end{array}$ & $1.156(0.563-2.980)$ & 0.739 \\
$\begin{array}{l}\text { Histopathological grade } \\
\quad(\text { Well + moderately/poorly) }\end{array}$ & $1.824(0.945-6.412)$ & 0.003 \\
$\begin{array}{l}\text { pStage } \\
\quad(\mathrm{I}-\mathrm{II} / \mathrm{III}-\mathrm{IV})\end{array}$ & $1.416(0.602-4.370)$ & 0.020 \\
$\begin{array}{l}\text { RCAS1 expression } \\
\quad(\text { Low/high })\end{array}$ & $1.484(0.622-4.580)$ & 0.008 \\
\hline
\end{tabular}

and multivariate analysis. These findings are in accordance with previous substantial evidence in carcinomas of other anatomic sites of the gastrointestinal tract, such as oral, esophageal and colorectal carcinomas [26-33]. Concerning gastric cancer, Nakamura et al. documented that RCAS1 staining pattern (diffuse or not) correlated with tumor size, depth of tumour invasion, histological type, and lymph node metastasis [15]. However, RCAS1 positivity was not associated with the clinicopathological variables examined, while no data concerning the patients' survival was reported [15]. In a study by Fukuda et al, which was performed on 129 T3 gastric carcinoma cases, RCAS1 expression was also significantly correlated with histological type and lymph node metastasis, as well as patients' prognosis [16]. In another study applying a semiquantitative RT-PCR analysis, RCAS1 mRNA levels in gastric adenocarcinoma tissues were significantly higher compared to nonneoplastic tissues [17]. The perinuclear pattern of RCAS1 expression was more frequently observed 


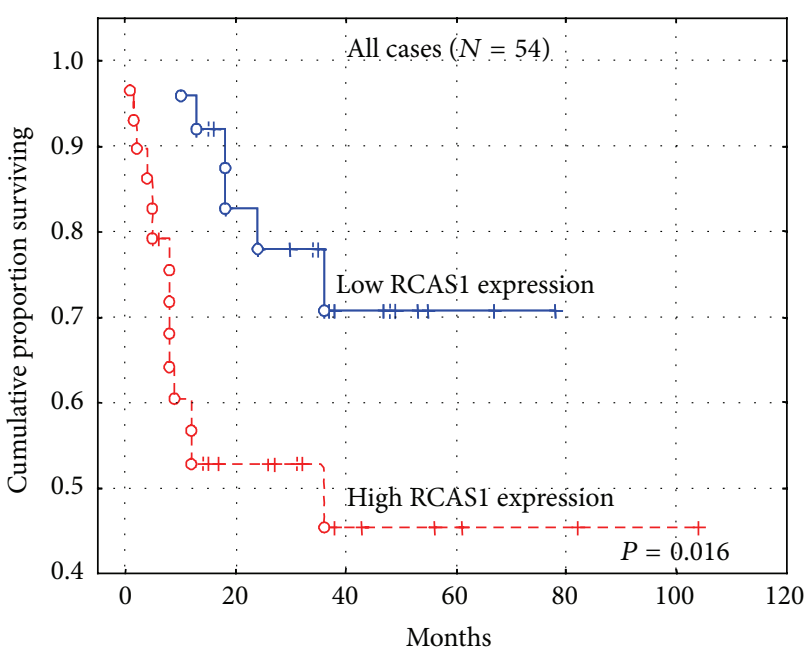

(a)

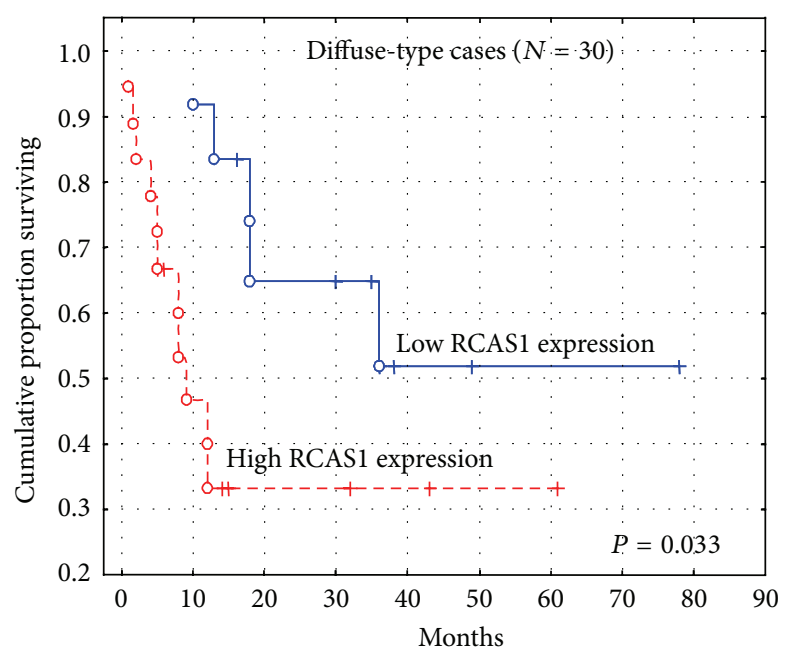

(b)

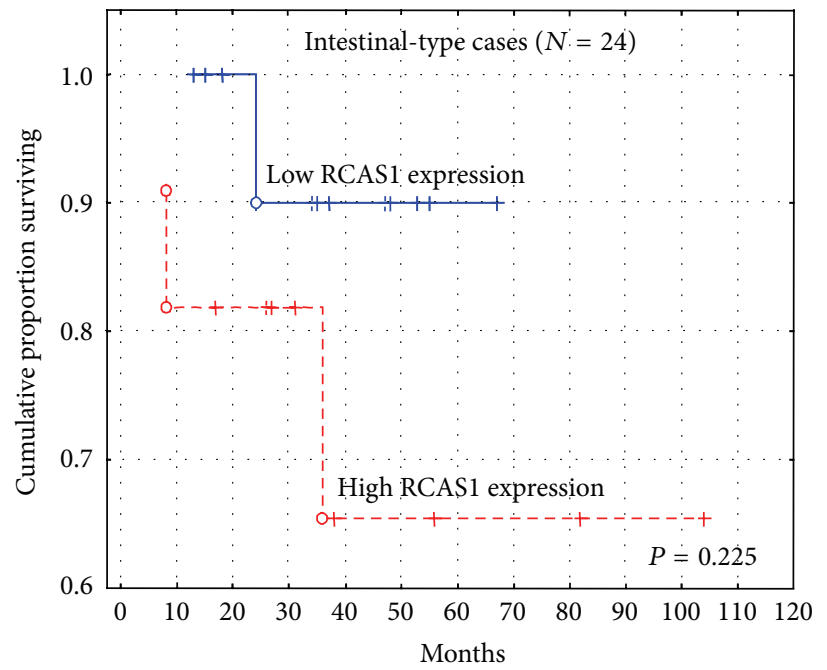

(c)

FIgURE 2: Kaplan-Meier survival analysis stratified according to RCAS1 expression in (a) all gastric cancer cases, (b) diffuse-type gastric cancer cases, and (c) intestinal-type gastric cancer cases.

in well differentiated gastric adenocarcinoma compared to moderate ones. The diffuse pattern of RCAS1 expression was more frequently recognized in gastric carcinomas which invaded beyond the submucosa compared to intramucosal carcinomas [17]. In contrast to the previous studies, we did not find any significant association with the presence of lymph node metastasis and the grade of differentiation; however, diffuse type gastric cancer cases showed an increased frequency of enhanced RCAS1 expression compared to intestinal ones without, though, reaching statistical significance.

Currently, substantial evidence has suggested that RCAS1 expression upregulation may play a crucial role in tumor progression by enabling cancer cells to evade immune surveillance $[3,34]$. Interestingly, RCAS1 induced apoptosis in both cultured human lymphoma cell lines and normal peripheral lymphocytes, which express RCAS1 receptor [3]. This apoptotic effect was supported to be mediated through induction of genes or caspase molecules, which strongly abrogated RCAS1-induced apoptosis, in vitro [3]. Moreover, RCAS1 facilitated tumor cell invasion of connective tissue in uterine cervical cancer via the enhancement of invasive potency by induction of stromal tissue remodeling, as well as through evasion of antitumor immune surveillance by an apoptotic counterattack mechanism against lymphocytes [23]. RCAS1 may also modulate surface expression of tumor-associated Olinked glycan structures, which are considered to participate in cell adhesion, invasion, and metastasis of cancer cells [35]. Taking into account the previous considerations, further studies should be performed in order to delineate whether RCAS1 positive tumor cells may induce apoptosis to their surrounding tumor infiltrating lymphocytes. As RCAS1 is easily detectable in biofluids $[36,37]$ further research effort should also be orientated to the determination of RCAS1 
levels in serum or saliva during and after the treatment of gastric cancer patients with the aim of recognizing patients' recurrence.

\section{Conclusions}

The present study supported evidence that RCAS1 was abundantly expressed in human gastric adenocarcinoma. RCAS1 expression was associated with clinicopathological parameters crucial for patients' management and prognosis. Of even more clinical significance are the data supporting the contribution of RCAS1 in the pathophysiological aspects of the disease that affect patients' survival. These findings may suggest an important potential role of RCAS1 in the biological mechanisms underlying the carcinogenic evolution of gastric adenocarcinoma. Further research conducted on larger cohorts and focused on each histopathological type separately that additionally concerned more sensitive techniques is strongly recommended in order to assess whether RCAS1 may be considered as a useful biomarker in gastric adenocarcinoma, evaluating also its therapeutic utility in gastric cancer chemoprevention and treatment.

\section{References}

[1] K. Sonoda, M. Nakashima, T. Kaku, T. Kamura, H. Nakano, and T. A. Watanabe, "A novel tumor-associated antigen expressed in human uterine and ovarian carcinomas," Cancer, vol. 77, pp. 1501-1509, 1996.

[2] K. Yamaguchi, M. Enjoji, M. Nakashima, M. Nakamuta, T. Watanabe, and M. Tanaka, "Novel serum tumor marker, RCAS1, in pancreatic diseases," World Journal of Gastroenterology, vol. 11, no. 33, pp. 5199-5202, 2005.

[3] M. Nakashima, K. Sonoda, and T. Watanabe, "Inhibition of cell growth and induction of apoptotic cell death by the human tumor-associated antigen RCAS1," Nature Medicine, vol. 5, no. 8, pp. 938-942, 1999.

[4] T. Matsushima, M. Nakashima, K. Oshima et al., "Receptor binding cancer antigen expressed on SiSo cells, a novel regulator of apoptosis of erythroid progenitor cells," Blood, vol. 98, no. 2, pp. 313-321, 2001.

[5] K. Sonoda, S. Miyamoto, T. Hirakawa et al., "Association between RCAS1 expression and microenvironmental immune cell death in uterine cervical cancer," Gynecologic Oncology, vol. 97, no. 3, pp. 772-779, 2005.

[6] K. Sonoda, S. Miyamoto, M. Nakashima, and N. Wake, "The biological role of the unique molecule RCAS1: a bioactive marker that induces connective tissue remodeling and lymphocyte apoptosis," Frontiers in Bioscience, vol. 13, no. 3, pp. 1106-1116, 2008.

[7] Q.-Y. He, Y. H. Cheung, S. Y. Leung, S. T. Yuen, K.-M. Chu, and J.-F. Chiu, "Diverse proteomic alterations in gastric adenocarcinoma," Proteomics, vol. 4, no. 10, pp. 3276-3287, 2004.

[8] D. Forman and V. J. Burley, "Gastric cancer: global pattern of the disease and an overview of environmental risk factors," Best Practice and Research, vol. 20, no. 4, pp. 633-649, 2006.

[9] P. Hohenberger and S. Gretschel, "Gastric cancer," The Lancet, vol. 362, no. 9380, pp. 305-315, 2003.

[10] T. Chiba, H. Marusawa, and T. Ushijima, "Inflammation-associated cancer development in digestive organs: mechanisms and roles for genetic and epigenetic modulation," Gastroenterol, vol. 143, pp. 550-563, 2012.

[11] H. Tsujimoto, S. Ono, T. Ichikura, Y. Matsumoto, J. Yamamoto, and K. Hase, "Roles of inflammatory cytokines in the progression of gastric cancer: friends or foes?" Gastric Cancer, vol. 13, no. 4, pp. 212-221, 2010.

[12] J. Zabaleta, "Multifactorial etiology of gastric cancer," Methods in Molecular Biology, vol. 863, pp. 411-435, 2012.

[13] C. Giaginis, A. Giagini, and S. Theocharis, "Receptor-binding cancer antigen expressed on SiSo cells (RCAS1): a novel biomarker in the diagnosis and prognosis of human neoplasia," Histology and Histopathology, vol. 24, no. 6, pp. 761-776, 2009.

[14] K. Sonoda, "Novel therapeutic strategies to target RCAS1, which induces apoptosis via ectodomain shedding," Histology and Histopathology, vol. 26, no. 11, pp. 1475-1486, 2011.

[15] Y. Nakamura, K. Yamazaki, S. Oizumi et al., "Expression of RCAS1 in human gastric carcinoma: a potential mechanism of immune escape," Cancer Science, vol. 95, no. 3, pp. 260-265, 2004.

[16] K. Fukuda, S. Tsujitani, Y. Maeta, K. Yamaguchi, M. Ikeguchi, and N. Kaibara, "The expression of RCAS1 and tumor infiltrating lymphocytes in patients with T3 gastric carcinoma," Gastric Cancer, vol. 5, no. 4, pp. 220-227, 2002.

[17] M. Kubokawa, M. Nakashima, T. Yao et al., "Aberrant intracellular localization of RCAS1 is associated with tumor progression of gastric cancer," International Journal of Oncology, vol. 19, no. 4, pp. 695-700, 2001.

[18] P. Lauren, "The two histological main types of gastric carcinoma: diffuse and so-called intestinal-type carcinoma," Acta Pathologica et Microbiologica Scandinavica, vol. 64, pp. 31-49, 1965.

[19] L. H. Sobin and C. Wittekind, TNM Classification of Malignant Tumors, John Wiley \& Sons, New York, NY, USA, 5th edition, 1997.

[20] C. Giaginis, D. Davides, A. Zarros et al., "Clinical significance of tumor-associated antigen RCAS1 expression in human pancreatic ductal adenocarcinoma," Digestive Diseases and Sciences, vol. 53, no. 6, pp. 1728-1734, 2008.

[21] S. Theocharis, J. Klijanienko, C. Giaginis et al., "RCAS1 expression in mobile tongue squamous cell carcinoma: an immunohistochemical study," Medical Science Monitor, vol. 17, no. 8, pp. BR228-BR234, 2011.

[22] C. Giaginis, N. Demetriou, P. Alexandrou et al., "Receptor-binding cancer antigen expressed on SiSo cells (RCAS1) expression in human benign and malignant thyroid lesions," Medical Science Monitor, vol. 18, no. 4, pp. BR123-BR129, 2012.

[23] K. Sonoda, S. Miyamoto, M. Nakashima, and N. Wake, "Receptor-binding cancer antigen expressed on SiSo cells induces apoptosis via ectodomain shedding," Experimental Cell Research, vol. 316, no. 11, pp. 1795-1803, 2010.

[24] Y. Han, W. Qin, and G. Huang, "Knockdown of RCAS1 expression by RNA interference recovers T cell growth and proliferation," Cancer Letters, vol. 257, no. 2, pp. 182-190, 2007.

[25] T. Kino and G. P. Chrousos, "Tumor-associated, estrogen receptor-related antigen EBAG9: linking intracellular vesicle trafficking, immune homeostasis, and malignancy," Molecular Interventions, vol. 9, no. 6, pp. 294-298, 2009.

[26] S. Tsujitani, T. Honboh, and N. Kaibara, "Immune evasion by RCAS1 in relation to the expression of cadherin family proteins in colorectal carcinoma," Biotherapy, vol. 17, no. 2, pp. 154-159, 2003. 
[27] S. Tsujitani, H. Saito, S. Oka et al., "Prognostic significance of RCAS1 expression in relation to the infiltration of dendritic cells and lymphocytes in patients with esophageal carcinoma," Digestive Diseases and Sciences, vol. 52, no. 2, pp. 549-554, 2007.

[28] S. Tsujitani, H. Saito, T. Honboh et al., "Prognostic significance of receptor-binding cancer antigen expressed on SiSo cells (RCAS1) expression in relation to cadherin expression in patients with colorectal carcinoma," Diseases of the Colon and Rectum, vol. 50, no. 8, pp. 1241-1249, 2007.

[29] T.-C. Tsai, C.-H. Yu, S.-J. Cheng, B.-Y. Liu, H.-M. Chen, and C.P. Chiang, "Expression of RCAS1 is significantly associated with the progression and prognosis of oral squamous cell carcinomas in Taiwan," Oral Oncology, vol. 44, no. 8, pp. 759-766, 2008.

[30] K. Okada, M. Nakashima, K. Komuta et al., "Expression of tumor-associated membrane antigen, RCAS1, in human colorectal carcinomas and possible role in apoptosis of tumorinfiltrating lymphocytes," Modern Pathology, vol. 16, no. 7, pp. 679-685, 2003.

[31] Y. Nakakubo, Y. Hida, M. Miyamoto et al., "The prognostic significance of RCAS1 expression in squamous cell carcinoma of the oesophagus," Cancer Letters, vol. 177, no. 1, pp. 101-105, 2002.

[32] H. Kato, M. Nakajima, N. Masuda et al., "Expression of RCAS1 in esophageal squamous cell carcinoma is associated with a poor prognosis," Journal of Surgical Oncology, vol. 90, no. 2, pp. 8994, 2005.

[33] M. Fukuda, A. Tanaka, A. Hamao, S. Suzuki, K. Kusama, and H. Sakashita, "Expression of RCAS1 and its function in human squamous cell carcinoma of the oral cavity," Oncology Reports, vol. 12, no. 2, pp. 259-267, 2004.

[34] T. Akashi, H. Oimomi, K.-I. Nishiyama et al., "Expression and diagnostic evaluation of the human tumor-associated antigen RCAS1 in pancreatic cancer," Pancreas, vol. 26, no. 1, pp. 49-55, 2003.

[35] A. Engelsberg, R. Hermosilla, U. Karsten, R. Schülein, B. Dörken, and A. Rehm, "The Golgi protein RCAS1 controls cell surface expression of tumor-associated O-linked glycan antigens," Journal of Biological Chemistry, vol. 278, no. 25, pp. 2299823007, 2003.

[36] S. Coban, H. Ozkan, S. Köklü et al., "The utility of serum receptor-binding cancer antigen expressed on SiSo cells in gastrointestinal tract cancers," Canadian Journal of Gastroenterology, vol. 20, pp. 593-596, 2006.

[37] C. Giaginis, A. Margeli, G. Kouraklis, A. Zira, G. Tsourouflis, and S. Theocharis, "Diagnostic and prognostic utility of serum receptor-binding cancer antigen expressed on SiSo cells (RCAS1) levels in colon cancer patients," International Journal of Biological Markers, vol. 24, no. 2, pp. 70-76, 2009. 




The Scientific World Journal
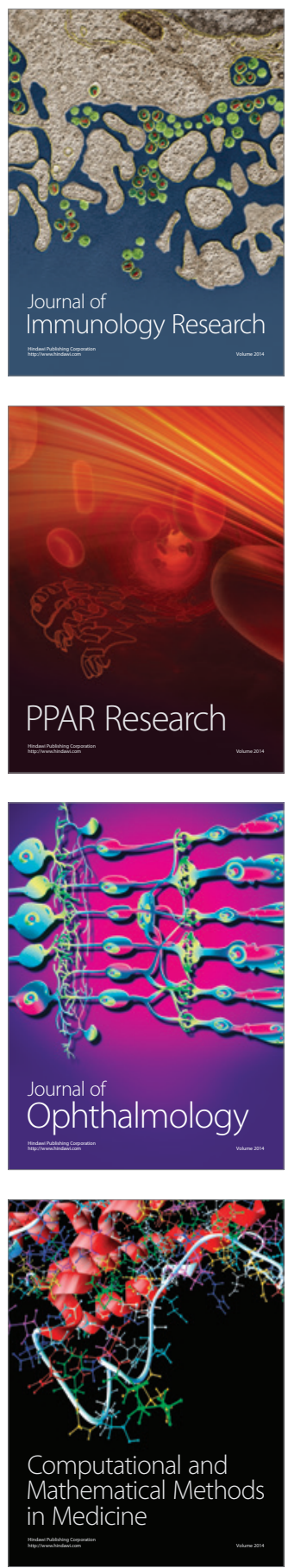

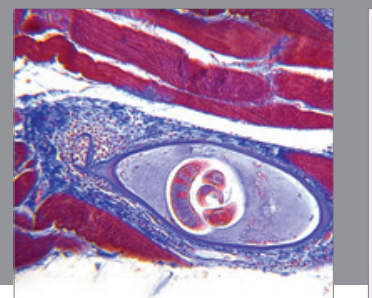

Gastroenterology

Research and Practice
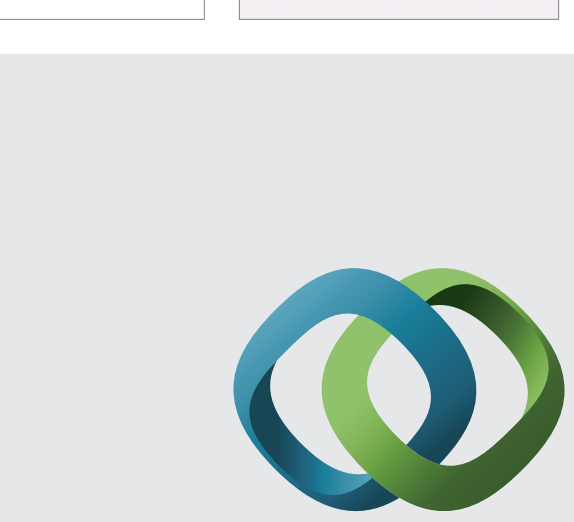

\section{Hindawi}

Submit your manuscripts at

http://www.hindawi.com
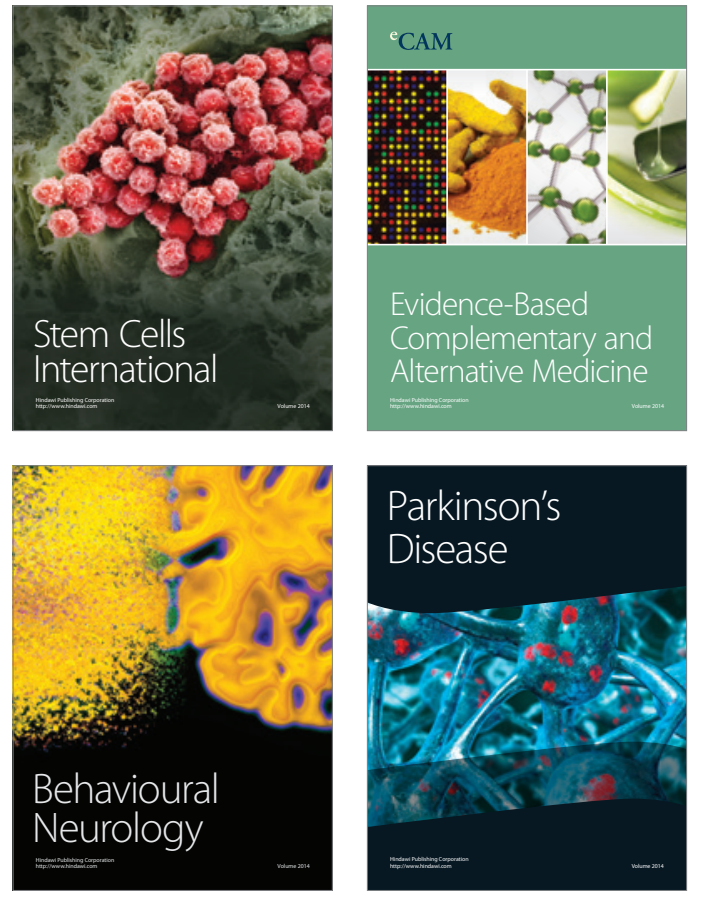
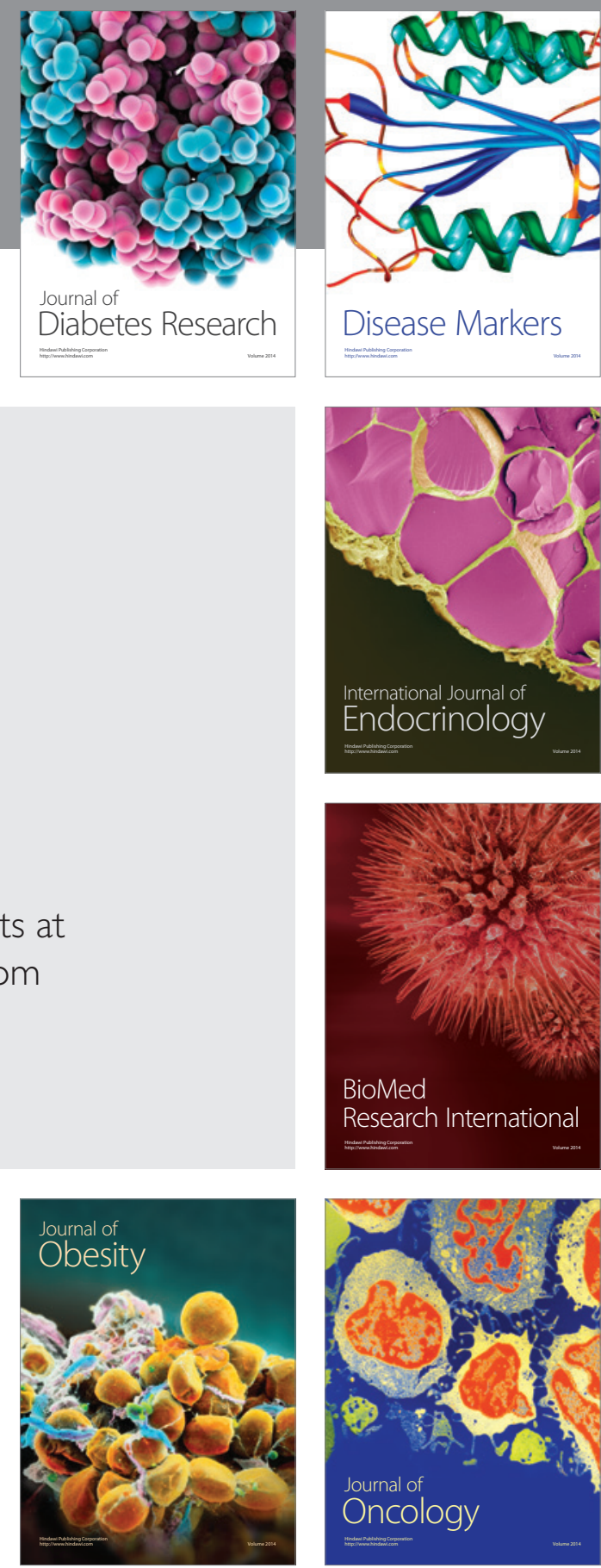

Disease Markers
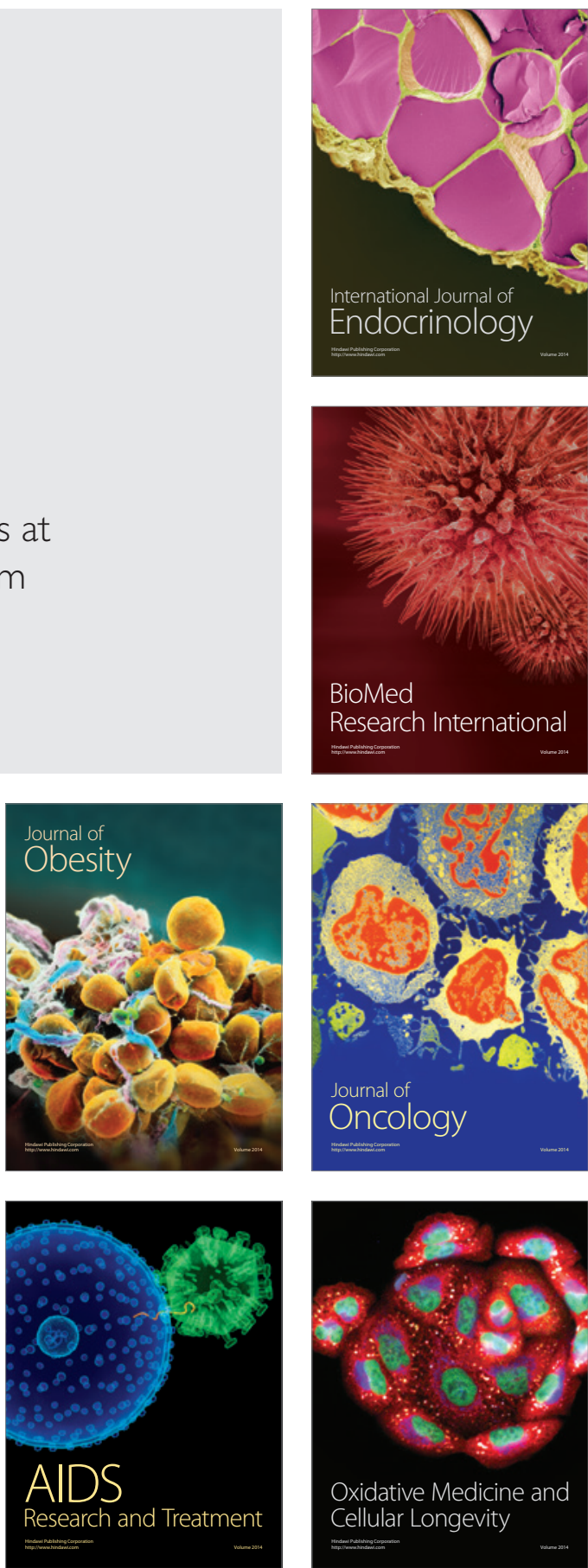